\title{
Chiral Polytopes from Hyperbolic Honeycombs
}

\author{
B. Nostrand ${ }^{1}$ and E. Schulte ${ }^{2}$ \\ ${ }^{1}$ Department of Mathematics and Statistics, York University, \\ 4700 Keele Street, North York, Ontario, Canada M3J 1P3 \\ nostrand@mathstat.yorku.ca \\ ${ }^{2}$ Department of Mathematics, Northeastern University, \\ 360 Huntington Avenue, Boston, MA 02115, USA \\ schulte@neu.edu
}

\begin{abstract}
Abstract polytopes are partially ordered structures which generalize the notion of polyhedra in a combinatorial sense. This concept includes all of the classical regular polytopes as well as many other well-known configurations. Chiral polytopes are abstract polytopes with maximal rotational symmetry which lack refiexive symmetry. We employ hyperbolic geometry to derive toroidal abstract polytopes of type $\{6,3,4\}$ and $\{6,3,5\}$ which are either regular or chiral. Their rotation groups are projective linear groups over finite rings.
\end{abstract}

\section{Introduction}

Regular polytopes have been studied since classical antiquity. In recent years there has been considerable interest in a class of combinatorial structures known as abstract polytopes [5], [9]-[13]. These abstract polytopes generalize the classical concept of a convex polytope to include somewhat more sophisticated geometric and topological structures. An abstract polytope is regular if it achieves maximal symmetry by reflection. Classification of these figures by both local and global topological type plays a major role in the theory of regular abstract polytopes. The spherical case was extensively studied by Coxeter [3]. Lately, McMullen and Schulte [9]-[13], among others, have paid particular attention to the toroidal case.

Recently, Schulte and Weiss [17], [18] have been studying abstract polytopes which exhibit chirality. Informally, a geometrical figure is "chiral" if it exhibits rotational symmetry, but is not symmetrical under (hyperplane) reflection. Chiral abstract polytopes are abstract polytopes with maximal (combinatorial) rotational 
symmetry which lack reflexive symmetry. These polytopes occur in two enantiomorphic (mirror image) forms. Examples of rank 3 chiral polytopes can be produced from irreflexible maps (tessellations) on surfaces [2], [4]. In higher ranks it is considerably more difficult to construct chiral polytopes. In terms of groups, this can be explained by the fact that in a Coxeter group the normal subgroups of the rotation (even) subgroup tend to be normal in the full group. In terms of polytopes, this means that if an abstract polytope already has maximal rotational symmetry, then it tends to admit enough (combinatorial) reflections to have maximal reflexive symmetry as well. Thus, chirality is a rather sporadic phenomenon. This is true even in rank 3 . While we can easily construct three families of chiral maps for genus 1 [4], the next higher genus for which there are chiral maps is genus 7 [6], [20].

For rank 4 we can employ hyperbolic geometry to find explicitly examples of chiral polytopes. These are constructed from three-dimensional regular hyperbolic honeycombs $\{p, q, r\}$ by using the well-known one-to-one correspondence between isometries in hyperbolic 3-space and complex Möbius transformations. More exactly, we use the technique for constructing chiral and regular polytopes first reported by Weiss [24] and further developed by Schulte and Weiss [18]. This technique represents the fundamental region of the symmetry group $[p, q, r]$ of the honeycomb $\{p, q, r\}$ as an "orthoscheme" in the complex inversive plane. This is a representation of the rotation group $[p, q, r]^{+}$of $\{p, q, r\}$ as a group of Möbius transformations over some ring, or, equivalently, as a group of $2 \times 2$ matrices over the same ring. Here we are mainly interested in types $\{6,3,4\}$ and $\{6,3,5\}$, in which case the rings are $\mathbb{Z}[\omega, \sqrt{2}]$ and $\mathbb{Z}[\omega, \tau]$, respectively. Here, $\omega=e^{2 \pi i / 3}$ is the cube root of unity, and $\tau=(1+\sqrt{5}) / 2$, the "golden ratio." When we pass to a suitable quotient ring $R$, we can "destroy" the reflections in the group, and thereby construct chiral polytopes of rank 4 which are of type $\{6,3,4\}$ or $\{6,3,5\}$. These polytopes have toroidal facets and octahedral or icosahedral vertex-figures. Their groups are projective linear groups over $R$. They are examples of locally toroidal polytopes, in the sense that all of the facets and vertex-figures are spherical or toroidal, with at least one being toroidal. Constructing such polytopes provides a partial solution to a long-standing problem of Grünbaum [7] which, in its most general form, calls for the classification of all locally toroidal polytopes which are either chiral or regular.

\section{Preliminaries}

For a detailed discussion of chiral polytopes we refer to [17], and for the theory of regular polytopes to [5] and [14].

An (abstract) polytope $\mathscr{P}$, or an $n$-polytope, is a partially ordered set with a strictly monotone rank function with range $\{-1,0, \ldots, n\}$. An element $F \in \mathscr{P}$ with $\operatorname{rank}(F)=j$ is called a $j$-face; typically $F_{j}$ indicates a $j$-face. The maximal chains of $\mathscr{P}$ are called flags. We require that $\mathscr{P}$ have a smallest $(-1)$-face $F_{-1}$, a greatest $n$-face $F_{n}$, and that each flag contains exactly $n+2$ faces. Furthermore, $\mathscr{P}$ should 
be strongly flag-connected; that is, any two flags $\Phi$ and $\Psi$ of $\mathscr{P}$ can be joined by a sequence of flags $\Phi=\Phi_{0}, \Phi_{1}, \ldots, \Phi_{k}=\Psi$, such that each $\Phi_{i-1}$ and $\Phi_{i}$ are adjacent in the sense that they differ by just one face, and $\Phi \cap \Psi \subset \Phi_{i}$ for each $i$. Finally, $\mathscr{P}$ should have the following homogeneity property: whenever $F<G$, $\operatorname{rank}(F)=j-1$, and $\operatorname{rank}(G)=j+1$, then there are exactly two $j$-faces $H$ with $F<H<G$.

If $F$ and $G$ are faces of $\mathscr{P}$ with $F \leq G$, we call $G / F:=\{H \mid F \leq H \leq G\}$ a section of $\mathscr{P}$. We do not distinguish between a face $F$ and the section $F / F_{-1}$, which is itself a polytope with the same rank as $F$. The faces of rank 0,1 , and $n-1$ are called vertices, edges, and facets, respectively. If $F$ is a face of a polytope $\mathscr{P}$, the section $F_{n} / F$ is called the co-face of $\mathscr{P}$ at $F$, or the vertex-figure of $\mathscr{P}$ at $F$ if $F$ is a vertex.

A polytope $\mathscr{P}$ is regular if its (automorphism) group $A(\mathscr{P})$ is transitive on the flags. The group $A(\mathscr{P})$ of a regular $n$-polytope $\mathscr{P}$ is generated by a set of involutions $\rho_{0}, \ldots, \rho_{n-1}$, where each $\rho_{j}$ is the unique automorphism which fixes all but the $j$-face of a designated base flag $\Phi=\left\{F_{-1}, F_{0}, F_{1}, \ldots, F_{n}\right\}$ of $\mathscr{P}$. These distinguished generators of $A(\mathscr{P})$ satisfy the relations

$$
\left(\rho_{j} \rho_{k}\right)^{p_{j k}}=1 \quad(j, k=0, \ldots, n-1)
$$

where $p_{j j}=1, p_{j k}=p_{k j}=: p_{j}$ if $k=j-1$, and $p_{j k}=2$ otherwise. The $p_{j}$ 's are given by the type $\left\{p_{1}, \ldots, p_{n-1}\right\}$ or "Schläfli symbol" of $\mathscr{P}$.

For any regular polytope $\mathscr{P}$, the group $A(\mathscr{P})$ and its generators $\rho_{j}$ satisfy the intersection property

$$
\left\langle\rho_{j} \mid j \in J\right\rangle \cap\left\langle\rho_{j} \mid j \in K\right\rangle=\left\langle\rho_{j} \mid j \in J \cap K\right\rangle \quad \text { for } \quad J, K \subseteq\{0, \ldots, n-1\} .
$$

A group $A$ generated by involutions $\rho_{0}, \ldots, \rho_{n-1}(n \geq 1)$ which satisfy both the relations (1) and the intersection property (2) is called a (string) C-group. If $A$ is a C-group, then $A$ is indeed the group of a regular polytope $\mathscr{P}$ with $\rho_{0}, \ldots, \rho_{n-1}$ as its distinguished generators. For a regular polytope $\mathscr{P}$, the rotations $\sigma_{j}=\rho_{j-1} \rho_{j}$ $(j=1, \ldots, n-1)$ generate the rotation subgroup $A^{+}(\mathscr{P})$ of $A(\mathscr{P})$. This subgroup can be of at most index 2 in $A(\mathscr{P})$. A polytope is directly regular when the index $\left[A(\mathscr{P}): A^{+}(\mathscr{P})\right]=2$. For rank 4 polytopes [17], such as those dealt with in the this paper, the intersection property (2) reduces to

$$
\begin{gathered}
\left\langle\sigma_{1}\right\rangle \cap\left\langle\sigma_{2}\right\rangle=1=\left\langle\sigma_{2}\right\rangle \cap\left\langle\sigma_{3}\right\rangle, \\
\left\langle\sigma_{1}, \sigma_{2}\right\rangle \cap\left\langle\sigma_{2}, \sigma_{3}\right\rangle=\left\langle\sigma_{2}\right\rangle .
\end{gathered}
$$

Thus the rotation subgroup is sufficient to determine whether a group $A$ satisfies the intersection property.

A polytope $\mathscr{P}$ is chiral if it exhibits maximal rotational symmetry, but lacks reflexive symmetry. In particular, $\mathscr{P}$ is not regular, but for some designated base flag $\Phi=\left\{F_{-1}, F_{0}, \ldots, F_{n}\right\}$ automorphisms $\sigma_{1}, \ldots, \sigma_{n-1}$ of $\mathscr{P}$ exist such that each 
$\sigma_{j}$ cyclically permutes consecutive $j$-faces of $\mathscr{P}$ in the rank 2 section $F_{j+1} / F_{j-2}$ while fixing all of the remaining faces in $\Phi \backslash\left\{F_{j-1}, F_{j}\right\}$. These elements $\sigma_{1}, \ldots, \sigma_{n-1}$ are the distinguished generators of $A(\mathscr{P})$ and (when suitably oriented) satisfy the relations

$$
\begin{aligned}
\sigma_{j}^{p_{j}} & =1 & (1 \leq j \leq n-1), \\
\left(\sigma_{j} \sigma_{j+1} \cdots \sigma_{k}\right)^{2} & =1 & (1 \leq j<k \leq n-1) .
\end{aligned}
$$

Thus, we define $A^{+}(\mathscr{P}):=A(\mathscr{P})$, indicating that each element of $A(\mathscr{P})$ is a "rotation." If a group $A$ is generated by "rotations" $\sigma_{1}, \sigma_{2}, \sigma_{3}$ which satisfy the relations in (4) as well as the intersection property (3), then $A$ is indeed the group of a chiral 4-polytope or the rotation subgroup of a directly regular 4-polytope. Specifically, $\mathscr{P}$ is directly regular if and only if an involutory group automorphism $\rho: A \rightarrow A$ exists such that

$$
\rho\left(\sigma_{1}\right)=\sigma_{1}^{-1}, \quad \rho\left(\sigma_{2}\right)=\sigma_{1}^{2} \sigma_{2}, \quad \text { and } \quad \rho\left(\sigma_{3}\right)=\sigma_{3}
$$

This is a special case of a more general theorem [17].

Each isomorphism type of a chiral polytope $\mathscr{P}$ occurs in two enantiomorphic or "mirror-image" forms, each corresponding to an orientation of $\mathscr{P}$ determined by the selection of the base flag $\Phi$ (in either of two orbits of flags). Technically, an oriented chiral or directly regular polytope $(\mathscr{P},\{\Phi\})$ is a chiral or directly regular polytope $\mathscr{P}$ together with a distinguished orbit $\{\Phi\}$ of flags under the action of the rotation group $A^{+}(\mathscr{P})$. For chiral polytopes there are two distinct orientations, but for regular polytopes the two forms can be canonically identified. In this paper we omit the qualification "oriented" and simply talk about chiral or directly regular polytopes whenever enantiomorphism is not important for what is currently under discussion.

However, enantiomorphism is central to our understanding of classes of chiral polytopes. Let $\mathscr{P}_{1}$ and $\mathscr{P}_{2}$ be $n$-polytopes. Recall that if $\mathscr{P}_{1}$ and $\mathscr{P}_{2}$ are regular, then $\left\langle\mathscr{P}_{1}, \mathscr{P}_{2}\right\rangle$ denotes the class of all regular $(n+1)$-polytopes $\mathscr{P}$ with facets isomorphic to $\mathscr{P}_{1}$ and vertex-figures isomorphic to $\mathscr{P}_{2}$. Each nonempty class $\left\langle\mathscr{P}_{1}, \mathscr{P}_{2}\right\rangle$ contains a universal polytope denoted by $\left\{\mathscr{P}_{1}, \mathscr{P}_{2}\right\}[16]$. This polytope is directly regular if $\mathscr{P}_{1}$ and $\mathscr{P}_{2}$ are both directly regular. For chiral polytopes the definition of classes is more subtle and involves dealing with the two enantiomorphic forms in which the polytopes can occur. More precisely, if $\mathscr{P}_{1}$ and $\mathscr{P}_{2}$ are oriented chiral or directly regular polytopes, then $\left\langle\mathscr{P}_{1}, \mathscr{P}_{2}\right\rangle^{\text {ch }}$ denotes the class of all oriented chiral $(n+1)$-polytopes $\mathscr{P}$ with oriented facets isomorphic to $\mathscr{P}_{1}$ and oriented vertex-figures isomorphic to $\mathscr{P}_{2}$. Again, if $\mathscr{P}_{1}$ or $\mathscr{P}_{2}$ is chiral and $\left\langle\mathscr{P}_{1}, \mathscr{P}_{2}\right\rangle^{\text {ch }}$ is nonempty, then the class contains a universal polytope denoted by $\left\{\mathscr{P}_{1}, \mathscr{P}_{2}\right\}^{\text {ch }}[17]$. Observe that if we change the orientation of both $\mathscr{P}_{1}$ and $\mathscr{P}_{2}$, then the orientation of every polytope in the class is changed and, therefore, that of the universal $\left\{\mathscr{P}_{1}, \mathscr{P}_{2}\right\}^{\text {ch }}$ as well. However, the classes are not obviously related if the orientation of only one polytope is changed. 


\section{Tessellating the Torus and Locally Toroidal Polytopes}

Although we are interested in a $\{6,3\}_{(b, c)}$ tessellation of the torus, we describe the relations in terms of its dual $\{3,6\}_{(b, c)}$ tessellation [4]. Consider the $\{6,3\}$ tessellation of the Euclidean plane and the groups $[3,6]=\left\langle\rho_{2}, \rho_{1}, \rho_{0}\right\rangle$ and $[3,6]^{+}=\left\langle\sigma_{2}, \sigma_{1}\right\rangle$, with generators occurring in reverse order. Then the translations

$$
X=\left(\rho_{2} \rho_{1} \rho_{0}\right)^{2}=\sigma_{2}^{-1} \sigma_{1} \sigma_{2} \sigma_{1}^{-1}, \quad Y=\left(\rho_{1} \rho_{0} \rho_{2}\right)^{2}=\sigma_{1}^{-1} \sigma_{2}^{-1} \sigma_{1} \sigma_{2}
$$

are easily seen to be unit translations along oblique axes with a $\pi / 3$ angle of inclination. The orbit of $(0,0)$ under $\langle X, Y\rangle$ is the set of vertices of $\{3,6\}$. In particular,

$$
X^{b} Y^{c}=\left(\sigma_{2}^{-1} \sigma_{1} \sigma_{2} \sigma_{1}^{-1}\right)^{b}\left(\sigma_{1}^{-1} \sigma_{2}^{-1} \sigma_{1} \sigma_{2}\right)^{c}
$$

translates the origin $(0,0)$ to the point $(b, c)$ with coordinates relative to the basis $X, Y$. Thus, the fundamental region of the group $\left\langle X^{b} Y^{c}, X^{-c} Y^{b+c}\right\rangle$ is the parallelogram

$$
(b, c),(0,0),(-c, b+c),(b-c, b+2 c)
$$

The tessellated torus $\{6,3\}_{(b, c)}$ is now constructed by identifying opposite sides of this parallelogram. (See Fig. 1.) In particular, $\{3,6\}_{(b, c)}$ is regular (reflexible) if and only if $b=0, c=0$, or $b=c$. Otherwise, it is chiral (irreflexible). The chiral maps $\{3,6\}_{(b, c)}$ and $\{3,6\}_{(c, b)}$ are enantiomorphic. Thus, there are infinitely many chiral maps on the 2-torus. However, chirality is a sporadic phenomenon for higher genus. In fact, the work of Garbe [6] and Sherk [20] shows that the next appearance of chirality is for surfaces of genus 7 .

A polytope $\mathscr{P}$ is locally toroidal if its facets and vertex-figures are tessellations of the 2-sphere (spherical) or the torus (toroidal) with at least one being in fact toroidal. Thus, in principle, chiral toroidal maps $\{6,3\}_{(b, c)}$ can be used to construct locally toroidal chiral 4-polytopes. However, they cannot be used as 3-faces of

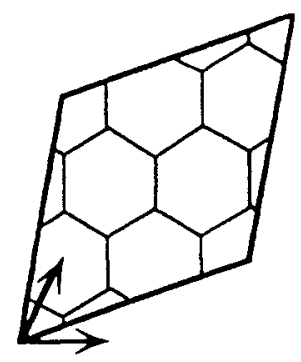

Fig. 1. $\{6,3\}_{(2,1)}$ tessellation of the torus. 
chiral 5-polytopes, because the 3-faces of any such polytope must be regular [17]. Recall that $\{6,3\}_{(b, c)}$ has $2 t$ vertices, $3 t$ edges, and $t$ hexagonal faces, where $t=b^{2}+b c+c^{2}$. The order of the rotation subgroup $[6,3]_{(b, c)}^{+}$is $6 t$. The number of facets, faces, edges, and vertices for a locally toroidal polytope $\mathscr{P}$ can be easily calculated from the order of its rotation group.

Proposition 1. If $\mathscr{P}$ is a locally toroidal 4-polytope in any of the classes

$$
\left\langle\{6,3\}_{(b, c)},\{3, r\}\right\rangle \text { and }\left\langle\{6,3\}_{(b, c)},\{3, r\}\right\rangle^{\text {ch }} \quad(r=3,4 \text {, or } 5)
$$

with rotation group $A^{+}(\mathscr{P})$, then $\mathscr{P}$ has

$$
\begin{array}{ll}
\text { Toroidal facets } & \left|A^{+}(\mathscr{P})\right| / 6 t, \\
\text { Hexagonal faces } & \left|A^{+}(\mathscr{P})\right| / 12, \\
\text { Edges } & \left|A^{+}(\mathscr{P})\right| / 2 r \\
\text { Vertices } & \left|A^{+}(\mathscr{P})\right| /\left|[3, r]^{+}\right|
\end{array}
$$

Later we also use the following lemma.

Lemma 1 [18, Lemma 12]. Let $m>1$ be an integer such that

$$
x^{2}+x+1 \equiv 0(m)
$$

is solvable. Let $\omega$ be a solution. Then a unique pair $b, c$ of positive integers satisfying

$$
m=b^{2}+b c+c^{2}, \quad(b, c)=1, \quad c \equiv \omega b(m)
$$

exists.

\section{Ring Extensions and Linear Groups}

Let $R$ be a commutative ring with identity 1 and let $G$ be a subgroup of the units $R^{*}$ of $R$. Let $L_{2}^{G}(R)$ denote the group of $2 \times 2$ matrices with entries in $R$ and determinants in $G$, and let $P L_{2}^{G}(R)$ denote the quotient group of $L_{2}^{G}(R)$ by its center $C_{2}^{G}(R)$. Note that $C_{2}^{G}(R)$ consists of all the matrices $\lambda I$ in $L_{2}^{G}(R)$ with $\lambda^{2} \in G$ (where $I$ denotes the identity matrix). Further, if $G=R^{*}$ we write $G L_{2}(R)$ and $P G L_{2}(R)$ for $L_{2}^{G}(R)$ and $P L_{2}^{G}(R)$, respectively, and if $G=1$, simply $S L_{2}(R)$ and $P S L_{2}(R)$.

Lemma 2 [23]. Let $R$ be a finite commutative ring with identity. Then $S L_{2}(R)$ is generated by all matrices of the form

$$
\left[\begin{array}{ll}
1 & a \\
0 & 1
\end{array}\right] \text { and }\left[\begin{array}{ll}
1 & 0 \\
b & 1
\end{array}\right] \text { with } a, b \in R
$$


Let $\omega:=e^{2 \pi i / 3}$, the complex cube root of unity with irreducible equation $\omega^{2}+\omega+1=0$. Let $\tau:=(1+\sqrt{5}) / 2$, the "golden ratio" with equation $\tau^{2}-$ $\tau-1=0$, and let $\sqrt{2}$ be the positive root of $x^{2}-2=0$. We are interested in the rings $\mathbb{Z}[\sqrt{2}]$ and $\mathbb{Z}[\omega]$ (the Eisenstein integers), $\mathbb{Z}[\tau], \mathbb{Z}[\omega, \sqrt{2}]$, and $\mathbb{Z}[\omega, \tau]$, and their quotients. The field extensions $\mathbb{Q}(\omega, \sqrt{2})$ and $\mathbb{Q}(\omega, \tau)$ of $\mathbb{Q}$ are the splitting fields of the polynomials $\left(x^{2}+x+1\right)\left(x^{2}-2\right)$ and $\left(x^{2}+x+1\right)\left(x^{2}-x-1\right)$ over $\mathbb{Q}$. Both extensions are of degree 4 . In particular, $\mathbb{Q}$-bases of $\mathbb{Q}(\omega, \sqrt{2})$ and $\mathbb{Q}(\omega, \tau)$ are given by $1, \omega, \sqrt{2}, \omega \sqrt{2}$ and $1, \omega, \tau, \omega \tau$, respectively. The rings $\mathbb{Z}[\omega, \sqrt{2}]$ and $\mathbb{Z}[\omega, \tau]$ are the $\mathbb{Z}$-spans of these bases. For each integer $m \geq 2$, let

$$
\begin{array}{rlrl}
\mathbb{Z}_{m} & :=\mathbb{Z} / m \mathbb{Z}, & \\
\mathbb{Z}_{m}[\kappa]: & =\mathbb{Z}[\kappa] / m \mathbb{Z}[\kappa] & & \text { if } \quad \kappa=\sqrt{2}, \omega, \text { or } \tau, \\
\mathbb{Z}_{m}[\omega, \kappa] & :=\mathbb{Z}[\omega, \kappa] / m \mathbb{Z}[\omega, \kappa] & & \text { if } \quad \kappa=\sqrt{2} \text { or } \tau .
\end{array}
$$

Note that we do not require the equations $x^{2}+x+1=0, x^{2}-x-1=0$, or $x^{2}-2=0$ to be irreducible over $\mathbb{Z}_{m}$. We slightly abuse notation and denote a ring element and its image under the canonical projection by the same symbol. Note also that $\langle-\omega\rangle=\{ \pm 1, \pm \omega, \pm \bar{\omega}\}$, with $\bar{\omega}=\omega^{2}$, is the group of units in $\mathbb{Z}[\omega]$. Observe that the projections $\mathbb{Z}[\kappa] \rightarrow \mathbb{Z}_{m}[\kappa]$ and $\mathbb{Z}[\omega, \kappa] \rightarrow \mathbb{Z}_{m}[\omega, \kappa]$ map units onto units. If $m=p$ is a prime with $p \equiv 2(3)$, then $x^{2}+x+1=0$ is irreducible over $\mathbb{Z}_{p}$ and $\mathbb{Z}_{p}[\omega] \cong G F\left(p^{2}\right)$, the Galois field with $p^{2}$ elements. Similarly, if $m=p$ is a prime with $p \equiv \pm 3(8)$, then $x^{2}-2$ is irreducible over $\mathbb{Z}_{p}$ and $\mathbb{Z}_{p}[\sqrt{2}] \cong G F\left(p^{2}\right)$. Also, if $m=p$ is an odd prime with $p=2,3(5)$, then $x^{2}-x-1=0$ is irreducible over $\mathbb{Z}_{p}$ and $\mathbb{Z}_{p}[\tau] \cong G F\left(p^{2}\right)$.

Over the rings $\mathbb{Z}[\omega], \mathbb{Z}[\omega, \sqrt{2}]$, and $\mathbb{Z}[\omega, \tau]$, complex conjugation defines an involutory ring automorphism. This automorphism can also be thought of as being induced by the conjugation map $\alpha+\beta \omega \mapsto \alpha+\beta \omega^{2}$ on $\mathbb{Z}[\omega]$. For the quotient rings $\mathbb{Z}_{m}[\omega], \mathbb{Z}_{m}[\omega, \sqrt{2}]$, and $\mathbb{Z}_{m}[\omega, \tau]$, this ring automorphism induces a "conjugation" map which is again an involutory automorphism.

\section{Representing Hyperbolic Groups}

The Poincare half-space model for hyperbolic 3-space shows that the inversive plane is the absolute of the hyperbolic space $\mathbb{H}^{3}[19]$. Therefore, reflection in a hyperbolic plane is equivalent to inversion across a circle in the inversive plane. This circle is the intersection of the reflecting hyperbolic plane with the absolute. Thus, a group of displacements in $\mathbb{H}^{3}$ is isomorphic to a group of Möbius transformations over $\mathbb{C}$.

The directly regular and chiral polytopes constructed in this paper are all finite and locally toroidal. They are derived by the method of Schulte and Weiss [18] from their corresponding universal polytope which is isomorphic to a regular honeycomb $\{p, q, r\}$ in $\mathbb{H}^{3}[1],[21]$. 
The symmetry group $[p, q, r]=\left\langle\rho_{0}, \rho_{1}, \rho_{2}, \rho_{3}\right\rangle$ of the honeycomb $\{p, q, r\}$ is a reflection group, where $\rho_{0}, \rho_{1}, \rho_{2}, \rho_{3}$ are reflections across the four bounding hyperplanes in the fundamental orthoscheme. Thus, $[p, q, r]$ can be represented by a group of Möbius transformations generated by inversions across four circles cutting one another at angles corresponding to those between the reflecting hyperplanes. The representations for the two hyperbolic honeycombs used in this paper were discovered by Weiss [24].

Let $\omega, \tau$, and $\sqrt{2}$ be as above. Then

$$
\begin{aligned}
& {[6,3,4]=\left\langle\rho_{0}(z)=\bar{z}, \rho_{1}(z)=-\overline{\omega z}, \rho_{2}(z)=\sqrt{2}-\bar{z}, \rho_{3}(z)=1 / \bar{z}\right\rangle,} \\
& {[6,3,5]=\left\langle\rho_{0}(z)=\bar{z}, \rho_{1}(z)=-\overline{\omega z}, \rho_{2}(z)=\tau-\bar{z}, \rho_{3}(z)=1 / \bar{z}\right\rangle .}
\end{aligned}
$$

Therefore,

$$
\begin{aligned}
& {[6,3,4]^{+}=\left\langle\sigma_{1}(z)=-\omega z, \sigma_{2}(z)=\omega^{2}(z-\sqrt{2}), \sigma_{3}(z)=\sqrt{2}-1 / z\right\rangle,} \\
& {[6,3,5]^{+}=\left\langle\sigma_{1}(z)=-\omega z, \sigma_{2}(z)=\omega^{2}(z-\tau), \sigma_{3}(z)=\tau-1 / z\right\rangle}
\end{aligned}
$$

with $\sigma_{j}=\rho_{j-1} \rho_{j}$. Below we represent the Möbius transformations $\sigma_{j}$ by matrices in the usual way [19]. The correspondence between matrices and Möbius transformations is one-to-one up to scalar multiplication:

$$
\frac{a z+b}{c z+d} \leftrightarrow\left[\begin{array}{ll}
a & b \\
c & d
\end{array}\right]
$$

\section{Type $\{6,3,4\}$}

The generators $\sigma_{1}, \sigma_{2}$, and $\sigma_{3}$ of the rotation group $[6,3,4]^{+}$can be represented as Möbius transformations with corresponding matrices in $G L_{2}(\mathbb{Z}[\omega, \sqrt{2}])$ :

$$
\sigma_{1}=\left[\begin{array}{cc}
-\omega & 0 \\
0 & 1
\end{array}\right], \quad \sigma_{2}=\left[\begin{array}{cc}
\omega^{2} & -\omega^{2} \sqrt{2} \\
0 & 1
\end{array}\right], \quad \sigma_{3}=\left[\begin{array}{rr}
\sqrt{2} & -1 \\
1 & 0
\end{array}\right] \text {. }
$$

The polytopes are now constructed by considering these matrices as matrices over certain quotient rings $R$ of $\mathbb{Z}[\omega, \sqrt{2}]$. These quotients are chosen in such a way that the subgroup of $\left\langle\sigma_{1}, \sigma_{2}\right\rangle$ becomes the group of a $\{6,3\}_{(b, c)}$ map on the torus. In particular, if the map is chiral, this local chirality forces global chirality for the whole polytope.

The matrices $\sigma_{1}, \sigma_{2}$ in (12) generate (up to scalar multiplication) the rotation group $[6,3]^{+}$of the hexagonal planar tessellation $\{6,3\}$. Its translation subgroup is generated by the two translations

$$
\tau_{1}=\sigma_{2}^{-1} \sigma_{1} \sigma_{2} \sigma_{1}^{-1}=\left[\begin{array}{cc}
1 & -\omega^{2} \sqrt{2} \\
0 & 1
\end{array}\right] \text { and } \tau_{2}=\sigma_{1}^{-1} \sigma_{2}^{-1} \sigma_{1} \sigma_{2}=\left[\begin{array}{cc}
1 & \omega \sqrt{2} \\
0 & 1
\end{array}\right]
$$


In particular,

$$
\tau_{1}^{b} \tau_{2}^{c}=\left[\begin{array}{cc}
1 & (c-\omega b) \omega \sqrt{2} \\
0 & 1
\end{array}\right]
$$

Equation (6) can be used to construct the toroidal map $\{6,3\}_{(b, c)}$. To do so, we need to find a quotient ring $R$ where $(c-\omega b) \omega \sqrt{2}=0$. However, $\omega^{3}=1$ and thus $\omega$ is a unit in $\mathbb{Z}[\omega, \sqrt{2}]$ and any quotient of $\mathbb{Z}[\omega, \sqrt{2}]$. Also, if $m$ is odd, $\sqrt{2}$ is a unit in any ring $\mathbb{Z}_{m}[\sqrt{2}]$ or $\mathbb{Z}_{m}[\omega, \sqrt{2}]$, and also in $\mathbb{Z}_{m}$ if $x^{2}-2 \equiv 0(m)$ has a solution. It follows that in such rings the equation reduces to $c-\omega b=0$. In particular, if $m$ is such that the equation $x^{2}+x+1 \equiv 0(m)$ has a solution, then we may regard $\omega$ as an element of $\mathbb{Z}_{m}$ and solve the equation $c-\omega b=0$ in $\mathbb{Z}_{m}$. This gives us facets of type $\{6,3\}_{(b, c)}$ with $b^{2}+b c+c^{2}=m$. Otherwise, the nontrivial solutions of $c-\omega b=0$ with $b, c \in \mathbb{Z}_{m}$ are $(b, c)=(m, 0)($ or $(b, c)=(0, m))$ and $(b, c)=(m, m)$. However, we can rule out facets of type $\{6,3\}_{(m, m)}$, since the order of the translation $\tau_{1}$ is $m$ rather than $3 m$ as it is for $\{6,3\}_{(m, m)}$. It follows that the facets are toroidal maps of type $\{6,3\}_{(m, 0)}$. This remains true even for arbitrary $\mathbb{Z}_{m}[\omega, \sqrt{2}]$. Then the equation reduces to $(c-\omega b) \sqrt{2}=0$, or $c \sqrt{2}-$ $b \omega \sqrt{2}=0$, which yields the same values for $(b, c)$.

In our constructions of polytopes, the matrices $\sigma_{1}, \sigma_{2}, \sigma_{3}$ in $G L_{2}(R)$ are usually taken modulo scalars. More exactly, this means that $\sigma_{1}, \sigma_{2}, \sigma_{3}$ are considered to be elements of $P G L_{2}(R)$, so that $\left\langle\sigma_{1}, \sigma_{2}, \sigma_{3}\right\rangle$ is a subgroup of $P G L_{2}(R)$. As elements in $G L_{2}(R), \sigma_{1}, \sigma_{2}, \sigma_{3}$ are in $L_{2}^{\langle-\omega\rangle}(R):=\left\{S \in G L_{2}(R) \mid \operatorname{det}(S) \in\langle-\omega\rangle\right\}$. We denote the image of $L_{2}^{\langle-\omega\rangle}(R)$ in $P G L_{2}(R)$ by $P L_{2}^{\langle-\omega\rangle}(R)$; that is, $L_{2}^{\langle-\omega\rangle}(R)$ modulo the center of $G L_{2}(R)$.

In order to construct a polytope from a rotation group it is necessary to demonstrate the intersection property (3).

Lemma 3. $\left\langle\sigma_{1}, \sigma_{2}, \sigma_{3}\right\rangle$ satisfies the intersection property over any quotient ring $R$ of $\mathbb{Z}[\omega, \sqrt{2}]$ occurring in (11) with $m \geq 3$.

Proof. Trivially, $\left\langle\sigma_{1}\right\rangle \cap\left\langle\sigma_{2}\right\rangle=\{1\}=\left\langle\sigma_{2}\right\rangle \cap\left\langle\sigma_{3}\right\rangle$. Consequently, what remains to be shown is that

$$
U:=\left\langle\sigma_{1}, \sigma_{2}\right\rangle \cap\left\langle\sigma_{2}, \sigma_{3}\right\rangle \subseteq\left\langle\sigma_{2}\right\rangle
$$

Since $\left\langle\sigma_{2}, \sigma_{3}\right\rangle \cong[3,4]^{+} \cong S_{4}$ and $\sigma_{2} \in U,|U|=3,6,12$, or 24 . First, notice that

$$
\sigma_{3} \notin\left\langle\sigma_{1}, \sigma_{2}\right\rangle \subseteq\left\{\left[\begin{array}{ll}
\alpha & \beta \\
0 & \delta
\end{array}\right]\right\},
$$




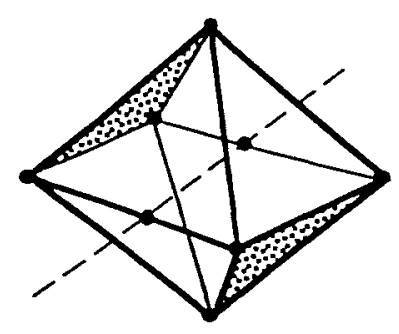

Fig. 2. The action of $\delta$ in $\{3,4\}$.

and therefore $|U| \neq 24$. Further, recall that $A_{4}$ is the only subgroup of $S_{4}$ of order 12 and contains all of the elements of order 3 . Now,

$$
\varphi:=\sigma_{3} \sigma_{2} \sigma_{3}^{-1}=\left[\begin{array}{cc}
2 \omega^{2}+1 & \omega \sqrt{2} \\
\omega^{2} \sqrt{2} & \omega+1
\end{array}\right] \notin\left\langle\sigma_{1}, \sigma_{2}\right\rangle
$$

because $\omega^{2} \sqrt{2} \neq 0$, but $\varphi$ is of order 3. Consequently, $|U| \neq 12$. Now consider the possibility that $|U|=6$. Since $U$ is a subgroup of $S_{4}$, the only possibility is $U \cong S_{3}$. As $U$ must contain [3] ${ }^{+}$, which is itself the rotation group of the base facet of the octahedron $\{3,4\}, U$ must be isomorphic to the group $\left\langle\sigma_{2}, \delta\right\rangle$ where $\delta$ is the half-turn which interchanges the base facet in the octahedron with its antipode. (See Fig. 2.) This rotation is given by

$$
\delta:=\sigma_{3}^{2} \sigma_{2} \sigma_{3}^{-1}=\left[\begin{array}{cc}
-\omega \sqrt{2} & -1+\omega \\
-1-2 \omega & \omega \sqrt{2}
\end{array}\right] \epsilon\left\langle\sigma_{2}, \sigma_{3}\right\rangle
$$

However, $\delta \notin\left\langle\sigma_{1}, \sigma_{2}\right\rangle$, and therefore $|U| \neq 6$.

Lemma 4. Let $m=2^{d} p_{1}^{e_{1}} \cdots p_{k}^{e_{k}}$ be the prime decomposition of $m$. Then $x^{2}+1 \equiv 0(m)$ is solvable if and only if $d=0,1$ and $p_{j} \equiv 1(4)$ for each $j=1, \ldots, k$.

Lemma 5. Let $m=p_{1}^{e_{1}} p_{2}^{e_{2}} \cdots p_{k}^{e_{k}}$ be the prime decomposition of $m$. Then $x^{2}-2 \equiv$ $0(m)$ and $x^{2}+x+1 \equiv 0(m)$ are both solvable if and only if the $p_{j} \equiv 1,7(24)$.

Proof. Let $m=2^{d} 3^{e} p_{1}^{e_{1}} \cdots p_{k}^{e_{k}}$ be the prime decomposition of $m$. Then $x^{2}+x+$ $1 \equiv 0(m)$ is solvable if and only if $d=0, e \in\{0,1\}$, and $p_{j} \equiv 1(3)$ for each $j=$ $1, \ldots, k$. Also, if $d=0$, then $x^{2}-2 \equiv O(m)$ is solvable if and only if $e=0$ and each $p_{j} \equiv \pm 1(8)[8]$. Consequently, combining the two conditions yields the result. 
Theorem 1. For each integer $m \geq 3$ there is a directly regular polytope $\mathscr{P}$ in $\left\langle\{6,3\}_{(m, 0)},\{3,4\}\right\rangle$ whose rotation group $A^{+}(\mathscr{P})$ is the subgroup of

$$
P L_{2}^{\langle-\omega\rangle}\left(\mathbb{Z}_{m}[\omega, \sqrt{2}]\right)
$$

generated by the matrices $\sigma_{1}, \sigma_{2}, \sigma_{3}$ in (12) over $\mathbb{Z}_{m}[\omega, \sqrt{2}]$.

Proof. The elements $\sigma_{1}, \sigma_{2}, \sigma_{3}$ have orders 6, 3, 4, respectively. By Lemma 3, $\left\langle\sigma_{1}, \sigma_{2}, \sigma_{3}\right\rangle$ has the intersection property and thus is the rotation group of a chiral or directly regular polytope $\mathscr{P}$ of type $\{6,3,4\}$. The conjugation on $\mathbb{Z}_{m}[\omega, \sqrt{2}]$ defined by $\omega \mapsto \omega^{2}$ induces an involutory group automorphism $\rho$ on $\left\langle\sigma_{1}, \sigma_{2}, \sigma_{3}\right\rangle$ such that $\rho\left(\sigma_{1}\right)=\sigma_{1}^{-1}, \rho\left(\sigma_{2}\right)=\sigma_{1}^{2} \sigma_{2}$, and $\rho\left(\sigma_{3}\right)=\sigma_{3}$. It follows that the polytope $\mathscr{P}$ is directly regular. As noted earlier, its facets are toroidal maps of type $\{6,3\}_{(m, 0)}$.

It would be interesting to identify the structure of the group in Theorem 1 at least where $m=p$ is a prime for which both $x^{2}+x+1$ and $x^{2}-2$ are irreducible modulo $p$. This occurs when $p \equiv 5,11(24)$. (See Lemma 5.) Then $\mathbb{Z}_{p}[\omega, \sqrt{2}]$ is a field of order $p^{4}$, and $P L_{2}^{\langle-\omega\rangle}\left(\mathbb{Z}_{p}[\omega, \sqrt{2}]\right)$ is isomorphic to $P S L_{2}\left(p^{4}\right)$ or $P G L_{2}\left(p^{4}\right)$.

Theorem 2. Let $m=p_{1}^{e_{1}} \cdots p_{k}^{e_{k}}$ where each $p_{j} \equiv 1,7(24)$, and let $b, c$ be positive integers such that $m=b^{2}+b c+c^{2},(b, c)=1$. Then a chiral polytope $\mathscr{P}$ in $\left\langle\{6,3\}_{(b, c)},\{3,4\}\right\rangle^{\text {ch }}$ exists such that

$$
A(\mathscr{P}) \cong \begin{cases}P S L_{2}\left(\mathbb{Z}_{m}\right) & \text { if every } p_{j} \equiv 1(24), \\ P S L_{2}\left(\mathbb{Z}_{m}\right) \ltimes C_{2} & \text { otherwise. }\end{cases}
$$

Proof. By our restrictions on $b$ and $c$, a unique $\omega \in \mathbb{Z}_{m}$ exists such that $\omega^{2}+\omega+1 \equiv 0(m)$ and $c \equiv \omega b(m)$. Let $\sqrt{2} \in \mathbb{Z}_{m}$ be a solution to $x^{2}-2 \equiv 0(m)$. (There are $2^{k}$ such solutions.) Now consider the generators $\sigma_{1}, \sigma_{2}, \sigma_{3}$ of (12) over $\mathbb{Z}_{m}$. Since $A:=\left\langle\sigma_{1}, \sigma_{2}, \sigma_{3}\right\rangle$ over $\mathbb{Z}_{m}$ is the homomorphic image of the rotation group of a hyperbolic honeycomb which preserves the periods of the generators, it is necessarily a rotation group of type $\{6,3,4\}$. Consequently, as the intersection property is known to hold, $A$ is the rotation group $A^{+}(\mathscr{P})$ of a chiral or directly regular polytope of type $\{6,3,4\}$. Now consider the generators $\sigma_{1}, \sigma_{2}, \sigma_{3}$ as matrices in

$$
L_{2}^{\langle-\omega\rangle}\left(\mathbb{Z}_{m}\right)=\left\{S \in G L_{2}\left(\mathbb{Z}_{m}\right) \mid \operatorname{det}(S) \in\langle-\omega\rangle\right\}
$$

Both $\omega$ and $\sqrt{2}$ are units in $\mathbb{Z}_{m}$. Consequently,

$$
B:=\sigma_{1}^{4} \sigma_{2} \sigma_{3}=\left[\begin{array}{rr}
0 & -1 \\
1 & 0
\end{array}\right] \text { and } U:=\left(\sigma_{1} \sigma_{2} \sigma_{1}^{3}\right)^{\kappa}=\left[\begin{array}{ll}
1 & 1 \\
0 & 1
\end{array}\right] \text {, }
$$


where $\kappa$ is the multiplicative inverse of $\sqrt{2}$. However, these two matrices generate $S L_{2}\left(\mathbb{Z}_{m}\right)$ and we have $\operatorname{det}\left(\sigma_{1}\right)=-\omega$. Hence, $\left\langle\sigma_{1}, \sigma_{2}, \sigma_{3}\right\rangle=L_{2}^{\langle-\omega\rangle}\left(\mathbb{Z}_{m}\right)$. We denote the center of $G L_{2}\left(\mathbb{Z}_{m}\right)$ by $K:=\left\{\lambda I \mid \lambda \in \mathbb{Z}_{m}^{*}\right\}$. Now, under the equivalence of Möbius transformations, we have the injective homomorphism

$$
\chi: P S L_{2}\left(\mathbb{Z}_{m}\right)=S L_{2}\left(\mathbb{Z}_{m}\right) /\left(K \cap S L_{2}\left(\mathbb{Z}_{m}\right)\right) \mapsto L_{2}^{\langle-\omega\rangle}\left(\mathbb{Z}_{m}\right) /\left(K \cap L_{2}^{\langle-\omega\rangle}\left(\mathbb{Z}_{m}\right)\right)=A
$$

However, $\chi$ is surjective if and only if $-\omega$ is a square. Since $\omega\left(=\omega^{4}\right)$ is a square, this is true if and only if -1 is a square modulo $m$. This implies, however, that $p \equiv 1(4)$ for each prime divisor $p$ of $m$. This proves the first case. If there is a prime $p$ with $p \not \equiv 1(4)$, then $A \cong P S L_{2}\left(\mathbb{Z}_{m}\right) \triangleright C_{2}$ with $C_{2}$ generated by $\sigma_{1}^{3}\left(K \cap L_{2}^{\langle-\omega\rangle}\left(\mathbb{Z}_{m}\right)\right)$. All that remains is to characterize the toroidal facets. Now the ring is $\mathbb{Z}_{m}$ and $\omega$ cannot be an independent basis element, but $c=b \omega$ and thus the toroidal figure $\{6,3\}_{(b, c)}$ must be chiral.

Example 1. Consider the case where $m=7,(b, c)=(1,2)$, and $\omega=2$. Then $A^{+}(\mathscr{P}) \cong P G L_{2}(7)$, of order 336 . Thus,

$\begin{array}{lr}\text { Toroidal facets } & 8, \\ \text { Hexagonal faces } & 28, \\ \text { Edges } & 42, \\ \text { Vertices } & 14 .\end{array}$

As the number of vertices in $\{6,3\}_{(1,2)}$ is also 14 , this is an example of a flat polytope. Recall that a polytope is flat if each of its facets shares the same set of vertices. See Fig. 3 for a picture of the graph of this polytope.

The Petrie polygon of a toroidal facet is a Hamiltonian cycle of the 14 vertices. One of these Hamiltonian cycles is immediately visible in Fig. 3. A hexagonal 2 -face of the corresponding facet can be found by enumerating the vertices along this Hamiltonian cycle from 0 to 13 and taking the "twisted" sequence $\{0,1,2,11,10,9\}$ as the boundary of one of the hexagonal 2 -faces of the toroidal facet. The remaining six 2 -faces of this facet can be found by taking adjacent

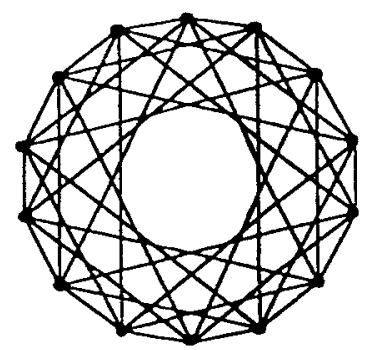

Fig. 3. The flat polytope in $\left\langle\{6,3\}_{(1,2)},\{3,4\}\right\rangle^{\text {ch }}$. 
hexagons clockwise around the Hamiltonian cycle. This is equivalent to adding $2(\bmod 14)$ to each of the vertices of successive 6-cycles.

Thus, we have found the seven hexagonal 2-faces of our toroidal "base facet." Each of these 2-faces is incident to a 2-face of a unique toroidal facet for seven new facets in all. Such a new facet can be found for the "base 2-face" by applying the permutation $(37513)(46128)$ to our Hamiltonian cycle. This produces a new Hamiltonian cycle which is then used to construct a new toroidal facet whose own base 2 -face is incident to our base 2 -face $\{0,1,2,11,10,9\}$. The remaining facets can be found by cyclically permuting the labels for our original Hamiltonian cycle and reapplying our technique. Thus, we can construct eight toroidal facets in all.

A further example illustrates our construction of chiral polytopes for composite values of $m$.

Example 2. If $m=217=7 \cdot 31$, then $m=8^{2}+8 \cdot 9+9^{2}=3^{2}+3 \cdot 13+13^{2}$. Now we have two polytopes $\mathscr{P}$ with groups $A(\mathscr{P}) \cong P S L_{2}\left(\mathbb{Z}_{217}\right) \bowtie C_{2}$, of order 4999680. One has facets of type $\{6,3\}_{(8,9)}$ and the other of type $\{6,3\}_{(3,13)}$. If we take the summands in the representation of $m$ in the reverse order, we arrive at polytopes which are isomorphic to these but are of the opposite enantiomorphic form, with facets $\{6,3\}_{(9,8)}$ and $\{6,3\}_{(13,3)}$, respectively. For either polytope, we have

$\begin{array}{lr}\text { Toroidal facets } & 3,840, \\ \text { Hexagonal faces } & 416,640, \\ \text { Edges } & 624,960, \\ \text { Vertices } & 208,320 .\end{array}$

In either case, each of the toroidal facets has 217 hexagonal faces. However, the two polytopes are clearly different.

Theorem 3. Let $p$ be a prime such that $p \equiv 13,19(24)$, and let $b, c$ be the unique pair of positive integers such that $p=b^{2}+b c+c^{2}, b>c$ and $(b, c)=1$. Then $a$ chiral polytope $\mathscr{P}$ in $\left\langle\{6,3\}_{(b, c)},\{3,4\}\right\rangle^{\text {ch }}$ with group

$$
A(\mathscr{P}) \cong P G L_{2}(p)
$$

exists.

Proof. By our assumptions about $p, x^{2}+x+1 \equiv 0(p)$ has a solution, but there is no solution for $x^{2}-2 \equiv 0(p)$. Choose $\omega \in \mathbb{Z}_{p}$ such that $\omega^{2}+\omega+1=0$ and $c=b \omega$. We now operate over the field $\mathbb{Z}_{p}[\sqrt{2}]$. Since the intersection property is known to hold, $A:=\left\langle\sigma_{1}, \sigma_{2}, \sigma_{3}\right\rangle$ over $\mathbb{Z}_{p}[\sqrt{2}]$ is the rotation group $A^{+}(\mathscr{P})$ of a 
chiral or directly regular polytope $\mathscr{P}$ of type $\{6,3,4\}$. Clearly, the generating matrices are in $G L_{2}\left(\mathbb{Z}_{p}[\sqrt{2}]\right)$ and therefore $A^{+}(\mathscr{P})$ is a subgroup of

$$
P G L_{2}\left(\mathbb{Z}_{p}[\sqrt{2}]\right) \cong P G L_{2}\left(p^{2}\right)
$$

The nontrivial subgroups of $P G L_{2}\left(p^{2}\right)$ are known to be isomorphic to one of the following groups:

(a) The dihedral groups of order $p^{2} \pm 1$ and their subgroups.

(b) A group $H$ of order $p^{2}\left(p^{2}-1\right) / 2$ and its subgroups.

(c) $A_{4}, S_{4}$, or $A_{5}$.

(d) $P S L_{2}(p), P G L_{2}(p), P S L_{2}\left(p^{2}\right)$, or $P G L_{2}\left(p^{2}\right)$.

Trivially, $A_{4}, S_{4}$, and $A_{5}$ are all too small to be $A^{+}(\mathscr{P})$. Also,

$$
\sigma_{1} \sigma_{2} \sigma_{1}^{3}=\left[\begin{array}{rr}
1 & \sqrt{2} \\
0 & 1
\end{array}\right]
$$

has order $p$ in $A^{+}(\mathscr{P})$, which rules out the groups in part (a). Now, in order for a group $H$, as in part (b), to contain $S_{4} \cong[3,4]^{+}, 48$ must divide $p^{2}-1$, and $p^{2} \equiv 1(48)$. However, $p \equiv 13,19(24)$ so this is impossible. What remains are the groups listed in part (d).

First we observe that, since $A^{+}(\mathscr{P})$ is generated by $\sigma_{1}, \sigma_{2}, \sigma_{3}$, we can write $A^{+}(\mathscr{P})=N \cdot\left\langle\sigma_{3}\right\rangle$ where $N$ is the normal closure of $\left\langle\sigma_{1}, \sigma_{2}\right\rangle$ in $A^{+}(\mathscr{P})$, the group generated by the conjugates of the elements in $\left\langle\sigma_{1}, \sigma_{2}\right\rangle$. Now the translation subgroup $T$ of $\left\langle\sigma_{1}, \sigma_{2}\right\rangle$ is generated by

$$
\tau_{1}=\sigma_{2}^{-1} \sigma_{1} \sigma_{2} \sigma_{1}^{-1}=\left[\begin{array}{cc}
1 & -\omega^{2} \sqrt{2} \\
0 & 1
\end{array}\right]
$$

(or, equivalently, by $\tau_{2}=\sigma_{1}^{-1} \sigma_{2}^{-1} \sigma_{1} \sigma_{2}$ ). However, $\left\langle\sigma_{1}, \sigma_{2}\right\rangle=\left\langle\sigma_{1}\right\rangle \cdot T$. Hence,

$$
\left\langle\sigma_{1}, \sigma_{2}\right\rangle=\left\{\left[\begin{array}{cc}
d & e \sqrt{2} \\
0 & 1
\end{array}\right] \mid d \in\langle-\omega\rangle, e \in \mathbb{Z}_{p}\right\}
$$

of order $6 p$. Clearly, $N$ is generated by the conjugates $\sigma_{3}^{j} \varphi \sigma_{3}^{-j}$ where $j=0, \ldots, 3$ and $\varphi \in\left\langle\sigma_{1}, \sigma_{2}\right\rangle$. It is now straightforward to check that each conjugate $\sigma_{3}^{j} \varphi \sigma_{3}^{-j}$ is contained in

$$
G:=\left\{\left[\begin{array}{cc}
d & e \sqrt{2} \\
f \sqrt{2} & g
\end{array}\right] \mid d, e, f, g \in \mathbb{Z}_{p}, d g-2 e f \neq 0\right\}
$$


$G$ is clearly a subgroup of $P G L_{2}\left(\mathbb{Z}_{p}[\sqrt{2}]\right)$ of order less than $p^{4}$. Hence, $N \subset G$ and $A^{+}(\mathscr{P})$ has order less than $4 p^{4}$. However, $\left|P S L_{2}\left(p^{2}\right)\right|=p^{2}\left(p^{4}-1\right) / 2>4 p^{4}$ and thus we can rule out the last two groups in part (d). Hence $A^{+}(\mathscr{P})$ is either $P S L_{2}(p)$ or $P G L_{2}(p)$.

Now $\sigma_{3} \notin G$ and thus $\sigma_{3} \notin N$. It follows that $N$ is a nontrivial proper normal subgroup of $A^{+}(\mathscr{P})$. This rules out the simple group $P S L_{2}(p)$, and therefore $A^{+}(\mathscr{P}) \cong P G L_{2}(p)$.

Theorem 4. For any $m=p_{1}^{e_{1}} \cdots p_{k}^{e_{k}}$ where each $p_{j} \equiv 1,7,17,23(24)$, with at least one $p_{j} \equiv 17,23(24)$, a directly regular polytope $\mathscr{P}$ in $\left\langle\{6,3\}_{(m, 0)},\{3,4\}\right\rangle$ exists such that

$$
A^{+}(\mathscr{P}) \cong \begin{cases}P S L_{2}\left(\mathbb{Z}_{m}[\omega]\right) & \text { if every } \quad p_{j} \equiv 1,17(24) \\ P S L_{2}\left(\mathbb{Z}_{m}[\omega]\right) \propto C_{2} & \text { otherwise. }\end{cases}
$$

Proof. By our assumptions about $m, x^{2}+x+1 \equiv 0(m)$ does not have a solution, but $x^{2}-2 \equiv 0(m)$ does. Let $\sqrt{2}$ denote a solution for $x^{2}-2 \equiv 0(m)$. We now operate over $\mathbb{Z}_{m}[\omega]$. Again, $A=\left\langle\sigma_{1}, \sigma_{2}, \sigma_{3}\right\rangle$ over $\mathbb{Z}_{m}[\omega]$ is the rotation group $A^{+}(\mathscr{P})$ of a chiral or directly regular polytope of type $\{6,3,4\}$. We now identify $A$ by considering the generators $\sigma_{1}, \sigma_{2}, \sigma_{3}$ as matrices in $G L_{2}\left(\mathbb{Z}_{m}[\omega]\right)$, or, more specifically, in

$$
L_{2}^{\langle-\omega\rangle}\left(\mathbb{Z}_{m}[\omega]\right)=\left\{S \in G L_{2}\left(\mathbb{Z}_{m}[\omega]\right) \mid \operatorname{det}(S) \in\langle-\omega\rangle\right\}
$$

Now $\sqrt{2}$ is a unit. Consequently,

$$
B:=\sigma_{1}^{4} \sigma_{2} \sigma_{3}=\left[\begin{array}{rr}
0 & -1 \\
1 & 0
\end{array}\right] \text { and } U:=\left(\sigma_{1} \sigma_{2} \sigma_{1}^{3}\right)^{\kappa}=\left[\begin{array}{ll}
1 & 1 \\
0 & 1
\end{array}\right] \text {, }
$$

where $\kappa$ is the multiplicative inverse of $\sqrt{2}$. Further,

$$
\sigma_{1}^{4} U \sigma_{1}^{2}=\left[\begin{array}{cc}
1 & \omega \\
0 & 1
\end{array}\right]
$$

These three matrices generate $S L_{2}\left(\mathbb{Z}_{m}[\omega]\right)$, and hence the matrices for $\sigma_{1}, \sigma_{2}, \sigma_{3}$ generate $L_{2}^{\langle-\omega\rangle}\left(\mathbb{Z}_{m}[\omega]\right)$. Therefore, consequently, $A=P L_{2}^{\langle-\omega\rangle}\left(\mathbb{Z}_{m}[\omega]\right)$. Now, $\omega$ is a square in $\mathbb{Z}_{m}[\omega]$, and thus $-\omega$ is a square if and only if -1 is a square. If $x=a+b \omega \in \mathbb{Z}_{m}[\omega]$ with $a, b \in \mathbb{Z}_{m}$, then $x^{2}=-1$ if and only if $a^{2}-b^{2} \equiv-1(m)$ and $(2 a-b) b \equiv 0(m)$. Clearly, if -1 is a quadratic residue modulo $m$, then -1 is a square (and we can take $b=0$ ). By Lemma 5 , our assumptions on $m$ require that this applies if and only if each $p_{j} \equiv 1,17(24)$. However, if $p_{j} \not \equiv 1,17(24)$ for at least one $j$, then -1 must be a nonsquare in $\mathbb{Z}_{m}[\omega]$. In fact, $x^{2}=-1$ implies that $(2 a-b) b \equiv O\left(p_{j}\right)$, and thus $b \equiv O\left(p_{j}\right)$ and $a^{2} \equiv-1\left(p_{j}\right)$; this is a contradiction. 
It follows that $P L_{2}^{\langle-\omega\rangle}\left(\mathbb{Z}_{m}[\omega]\right)$ is isomorphic to $P S L_{2}\left(\mathbb{Z}_{m}[\omega]\right)$ if each $p_{j} \equiv 1,17(24)$; and to $P S L_{2}\left(\mathbb{Z}_{m}[\omega]\right) \ltimes C_{2}$ otherwise, with the $C_{2}$ factor generated by the coset of $\sigma_{1}$ (whose determinant is the nonsquare $-\omega$ ).

Thus, all that remains is to characterize the toroidal facets and find the involutive group isomorphism $\rho$. As $\omega$ is an independent basis element, $\rho$ is induced by the ring automorphism $\omega \mapsto \bar{\omega}=\omega^{2}$. This involution clearly satisfies (5). Consequently, the resulting polytope is directly regular. As we saw in the discussion at the beginning of the section, the toroidal facets must be of type $\{6,3\}_{(m, 0)}$.

Corollary 1. Let $p$ be a prime with $p \equiv 17,23(24)$. Then a directly regular polytope $\mathscr{P}$ in $\left\langle\{6,3\}_{(p, 0)},\{3,4\}\right\rangle$ exists such that

$$
A^{+}(\mathscr{P}) \cong \begin{cases}P S L_{2}\left(p^{2}\right) & \text { if } p \equiv 17(24) \\ P G L_{2}\left(p^{2}\right) & \text { otherwise. }\end{cases}
$$

Proof. Now $\mathbb{Z}_{p}[\omega]$ is a field of order $p^{2}$. Also, $P G L_{2}(q) \cong P S L_{2}(q) \triangleright C_{2}$ for each $q=p^{k}$

\section{Type $\{6,3,5\}$}

The generators $\sigma_{1}, \sigma_{2}$, and $\sigma_{3}$ of the rotation group $[6,3,5]^{+}$can be represented as Möbius transformations with corresponding matrices in $G L_{2}(\mathbb{Z}[\omega, \tau])$ :

$$
\sigma_{1}=\left[\begin{array}{cc}
-\omega & 0 \\
0 & 1
\end{array}\right], \quad \sigma_{2}=\left[\begin{array}{cc}
\omega^{2} & -\omega^{2} \tau \\
0 & 1
\end{array}\right], \quad \sigma_{3}=\left[\begin{array}{cc}
\tau & -1 \\
1 & 0
\end{array}\right]
$$

Again, $\left\langle\sigma_{1}, \sigma_{2}\right\rangle=[6,3]^{+}$, and its translation subgroup is generated by

$$
\tau_{1}=\sigma_{2}^{-1} \sigma_{1} \sigma_{2} \sigma_{1}^{-1}=\left[\begin{array}{cc}
1 & -\omega^{2} \tau \\
0 & 1
\end{array}\right] \text { and } \tau_{2}=\sigma_{1}^{-1} \sigma_{2}^{-1} \sigma_{1} \sigma_{2}=\left[\begin{array}{cc}
1 & \omega \tau \\
0 & 1
\end{array}\right]
$$

In particular,

$$
\tau_{1}^{b} \tau_{2}^{c}=\left[\begin{array}{cc}
1 & \left(\omega c-\omega^{2} b\right) \tau \\
0 & 1
\end{array}\right]
$$

Given our translation in the plane (6), we know that to construct $\{6,3\}_{(b, c)}$ we need only find a quotient ring of $\mathbb{Z}[\omega, \tau]$ where $(c-\omega b) \omega \tau=0$. However, $\tau^{2}-\tau-1=0$ implies that $\tau(\tau-1)=1$, so both $\tau$ and $\omega$ are units in any quotient ring of $\mathbb{Z}[\omega, \tau]$. It follows that the equation reduces to $c-b \omega=0$. Consequently, if the transformations are represented by matrices over a finite ring $\mathbb{Z}_{m}$ in which $x^{2}+x+1=0$ finds a solution, then the identification is twisted and the toroidal map is of type $\{6,3\}_{(b, c)}$ where $b^{2}+b c+c^{2}=m$. Otherwise, the toroidal map is 
of type $\{6,3\}_{(m, 0)}$. The case where $\{6,3\}_{(m, m)}$ can be ruled out, because $\tau_{1}$ has order $m$ rather than $3 m$. In order to construct a polytope from a rotation group, it is necessary to demonstrate the intersection property.

Lemma 6. $\left\langle\sigma_{1}, \sigma_{2}, \sigma_{3}\right\rangle$ satisfies the intersection property over any quotient ring $R$ of $\mathbb{Z}[\omega, \tau]$ occurring in (11) with $m \geq 3$.

Proof. First, directly observe that $\left\langle\sigma_{1}\right\rangle \cap\left\langle\sigma_{2}\right\rangle=\{1\}=\left\langle\sigma_{2}\right\rangle \cap\left\langle\sigma_{3}\right\rangle$. Consequently, what remains to be shown is that

$$
U:=\left\langle\sigma_{1}, \sigma_{2}\right\rangle \cap\left\langle\sigma_{2}, \sigma_{3}\right\rangle \subseteq\left\langle\sigma_{2}\right\rangle
$$

Recall that $[3,5]^{+} \cong A_{5}$ and that $A_{5}$ is simple. In particular, $\left\langle\sigma_{2}, \sigma_{3}\right\rangle \cong A_{5}$. Now, $|U|$ must divide 60 and must itself be divisible by 3 . Consequently, $|U|=3,6,12$, 15,30 , or 60 . First, notice that

$$
\sigma_{3} \notin\left(\sigma_{1}, \sigma_{2}\right\rangle \subseteq\left\{\left[\begin{array}{ll}
\alpha & \beta \\
0 & \delta
\end{array}\right]\right\}
$$

and therefore $|U| \neq 60$. Also, $A_{5}$ does not have a subgroup of order 15 or 30 , and its subgroups of order 6 are isomorphic to $D_{3}$. However, $U$ contains the rotation group $\left\langle\sigma_{2}\right\rangle$ of a facet of $\{3,5\}$, and hence, if $|U|=6$, this forces $U=\left\langle\sigma_{2}, \delta\right\rangle$ with $\delta$ being a half-turn around an axis parallel to this facet (as in Fig. 2.) In particular, $\delta=\sigma_{3}^{2} \sigma_{2}\left(\sigma_{2} \sigma_{3}\right) \sigma_{2}^{-1} \sigma_{3}^{-2}=\left(\sigma_{3}^{2} \sigma_{2}^{2}\right)^{2} \sigma_{3} \sigma_{2}$. However, an easy computation shows that $\delta$ cannot be represented by an upper triangular matrix, hence $\delta \notin\left\langle\delta_{1}, \delta_{2}\right\rangle$. This rules out the case where $|U|=6$. Finally, every subgroup of $A_{5}$ which has order 12 is isomorphic to $A_{4} \cong[3,3]^{+}$. Consequently, only the rotation group of a tetrahedron inscribed in the vertex figure $\{3,5\}$ need be considered.

There are two possible ways to inscribe a tetrahedron $\{3,3\}$ into $\{3,5\}$ so that one vertex of $\{3,3\}$ is at the center of the base 2 -face of $\{3,5\}$. (That is, the symmetry group of the tetrahedron contains $\sigma_{2}$.) The rotation groups for these two tetrahedra are the subgroups $\left\langle\sigma_{2}, \varphi\right\rangle$ and $\left\langle\sigma_{2}, \psi\right\rangle$ of $[3,5]^{+}$where $\varphi$ and $\psi$ are rotations of 2 -faces of $\{3,5\}$ whose center is a vertex of the tetrahedron. Possible choices are

$$
\begin{array}{ll}
\varphi=\alpha \sigma_{2} \alpha^{-1}, & \alpha=\sigma_{2} \sigma_{3}^{2} \sigma_{2}^{-1} \sigma_{3}, \\
\psi=\beta \sigma_{2} \beta^{-1}, & \beta=\sigma_{2} \sigma_{3} \sigma_{2}^{-1} \sigma_{3}^{2} .
\end{array}
$$

Now

$$
\varphi=\left[\begin{array}{cc}
\omega^{2} \tau & -\tau(\omega \tau+1) \\
\tau-\omega^{2} & \omega^{2}(\tau-1)
\end{array}\right] \text { and } \psi=\left[\begin{array}{cc}
2 \tau-\omega\left(\tau^{2}+1\right) & \tau^{2}+\tau \\
-\omega \tau & -1
\end{array}\right]
$$


These matrices are not upper triangular, because $\tau-\omega^{2} \neq 0$ and $-\omega \tau \neq 0$. In fact, $-\omega \tau \neq 0$, because $\omega$ and $\tau$ are units. Also, $\tau-\omega^{2}=0$ would imply that $\tau^{2}+\tau+1=0=\tau^{2}-\tau-1$, and, thus, $2(\tau+1)=0$. This would force the quotient ring to be $\mathbb{Z}_{m}$ with $m$ even and $\tau=m / 2-1$, contradicting $\tau^{2}-\tau-1=0$. It follows that $\varphi, \psi \notin\left\langle\sigma_{1}, \sigma_{2}\right\rangle$. This excludes the case where $|U|=12$ and proves that $|U|=3$, as required.

Theorem 5. For each integer $m \geq 3$, there is a directly regular polytope $\mathscr{P}$ in $\left\langle\{6,3\}_{(m, 0)},\{3,5\}\right\rangle$ whose rotation group $A^{+}(\mathscr{P})$ is the subgroup of $P L_{2}^{\langle-\omega\rangle}\left(\mathbb{Z}_{m}[\omega, \tau]\right)$ generated by the matrices $\sigma_{1}, \sigma_{2}, \sigma_{3}$ in $(16)$ over $\mathbb{Z}_{m}[\omega, \tau]$.

Proof. When $m \geq 3$, the elements $\sigma_{1}, \sigma_{2}, \sigma_{3}$ have orders 6, 3, 5, respectively. Thus, as the intersection property has been shown to hold, $A:=\left\langle\sigma_{1}, \sigma_{2}, \sigma_{3}\right\rangle$ is the rotation group of a chiral or directly regular polytope $\mathscr{P}$ of type $\{6,3,5\}$. It is easy to verify that the ring automorphism defined by $\omega \mapsto \omega^{2}$ induces an involutory group automorphism $\rho$ on $A$ such that $\rho\left(\sigma_{1}\right)=\sigma_{1}^{-1}, \rho\left(\sigma_{2}\right)=\sigma_{1}^{2} \sigma_{2}$, and $\rho\left(\sigma_{3}\right)=\sigma_{3}$. It follows that $\mathscr{P}$ is directly regular and has facets of type $\{6,3\}_{(m, 0)}$.

As for Theorem 1, we do not know in general the structure of this group. Note that if $m=p$ is a prime for which both $x^{2}+x+1$ and $x^{2}-x-1$ are irreducible modulo $p$, then $\mathbb{Z}_{p}[\omega, \tau]$ is a field of order $p^{4}$ and $P L_{2}^{\langle-\omega\rangle}\left(\mathbb{Z}_{m}[\omega, \tau]\right)$ becomes $P S L_{2}\left(p^{4}\right)$ or $P G L_{2}\left(p^{4}\right)$.

Lemma 7. Let $m=2^{d} 3^{c} 5^{f} p_{1}^{e_{1}} p_{2}^{e_{2}} \cdots p_{k}^{e_{k}}$ be the prime decomposition of $m$. Then $x^{2}-x-1 \equiv O(m)$ is solvable if and only if $d=0, e=0, f=0,1$, and $p_{j} \equiv \pm 1(5)$ for each $j=1, \ldots, k$.

Proof. Since $x^{2}-x-1 \equiv 0(m)$ has no solution for $m=2,3$, necessarily $d=$ $e=0$, and if $d=e=0$, then $x^{2}-x-1 \equiv 0(m)$ is equivalent to $(2 x-1)^{2} \equiv 5(m)$. This forces $f=0,1$. Now 5 is a quadratic residue modulo $p_{j}^{e_{j}}$ if and only if $p_{j} \equiv \pm 1(5)$.

Lemma 8. Let $m=2^{d} 3^{c} 5^{f} p_{1}^{e_{1}} p_{2}^{e_{2}} \cdots p_{k}^{e_{k}}$ be the prime decomposition of $m$. Then $x^{2}+x+1 \equiv 0(m)$ and $x^{2}-x-1 \equiv 0(m)$ are both solvable if and only if $p_{j} \equiv 1$, $4(15)$ for $e a c h j=1, \ldots, k$.

Proof. If $m=2^{d} 3^{e} p_{1}^{e_{1}} \cdots p_{k}^{e_{k}}$ is the prime factorization of $m$, then $x^{2}+x+1 \equiv 0(m)$ is solvable if and only if $d=0, e=0,1$, and $p_{j} \equiv 1(3)$ for each $j=1, \ldots, k$. The result follows from this fact and the preceding lemma [18].

Theorem 6. Let $m=p_{1}^{e_{1}} \cdots p_{k}^{e_{k}}$ where each $p_{j} \equiv 1,4(15)$, and let $b, c$ be positive integers such that $m=b^{2}+b c+c^{2},(b, c)=1$. Then a chiral polytope $\mathscr{P}$ in 
$\left\langle\{6,3\}_{(b, c)},\{3,5\}\right\rangle^{\text {ch }}$ exists such that

$$
A(\mathscr{P}) \cong \begin{cases}P S L_{2}\left(\mathbb{Z}_{m}\right) & \text { if every } p_{j} \equiv 1,49(60), \\ P S L_{2}\left(\mathbb{Z}_{m}\right) \propto C_{2} & \text { otherwise. }\end{cases}
$$

Proof. By our restrictions on $b$ and $c$, a unique $\omega \in \mathbb{Z}_{m}$ exists such that $\omega^{2}+\omega+1 \equiv 0(m)$ and $c \equiv \omega b(m)$. Let $\tau \in \mathbb{Z}_{m}$ be such that $\tau^{2}-\tau-1 \equiv 0(m)$. (There are $2^{k}$ such choices for $\tau$.) Now consider the generators $\sigma_{1}, \sigma_{2}, \sigma_{3}$ of (16) over $\mathbb{Z}_{m}$. Again, $A$ is the rotation group $A^{+}(\mathscr{P})$ of a chiral or directly regular polytope of type $\{6,3,5\}$. Now consider the generators $\sigma_{1}, \sigma_{2}, \sigma_{3}$ as matrices in

$$
L_{2}^{\langle-\omega\rangle}\left(\mathbb{Z}_{m}\right)=\left\{S \in G L_{2}\left(\mathbb{Z}_{m}\right) \mid \operatorname{det}(S) \in\langle-\omega\rangle\right\}
$$

Both $\omega$ and $\tau$ are units in $\mathbb{Z}_{m}$. Consequently,

$$
B:=\sigma_{1}^{4} \sigma_{2} \sigma_{3}=\left[\begin{array}{rr}
0 & -1 \\
1 & 0
\end{array}\right] \text { and } U:=\left(\sigma_{1} \sigma_{2} \sigma_{1}^{3}\right)^{\kappa}=\left[\begin{array}{ll}
1 & 1 \\
0 & 1
\end{array}\right] \text {, }
$$

where $\kappa$ is the multiplicative inverse of $\tau$. Now we can complete our argument as in the proof for Theorem 2. In particular, the matrices generate $L_{2}^{\langle-\omega\rangle}\left(\mathbb{Z}_{m}\right)$ and $A \cong P S L_{2}\left(\mathbb{Z}_{m}\right)$ if -1 is a square modulo $m$. Otherwise, $A \cong P S L_{2}\left(\mathbb{Z}_{m}\right) \ltimes C_{2}$. However, -1 is a square modulo $m$ if and only if each $p_{j} \equiv 1(4)$. That is, $p_{j} \equiv 1$, $49(60)$.

The following lemma is a special case of a more general result on the conjugacy of certain subgroups of $S L_{2}(q)$ [23, p. 405].

Lemma 9. Let $G$ be a subgroup of $S L_{2}\left(p^{2}\right)$ of order ip $\left(p^{2}-1\right)$ with $i=1,2(p \neq 2)$. If $i=1$, then $G$ is conjugate in $G L_{2}\left(p^{2}\right)$ to the standard $S L_{2}(p)$ embedded in $S L_{2}\left(p^{2}\right)$. If $i=2$, then $G$ is conjugate in $G L_{2}\left(p^{2}\right)$ to the subgroup

$$
L:=\left\langle S L_{2}(p),\left[\begin{array}{cc}
\mu & 0 \\
0 & \mu^{-1}
\end{array}\right]\right\rangle
$$

of $S L_{2}\left(p^{2}\right)$, where the subgroup on the left is the standard $S L_{2}(p)$ and $\mu \in G F\left(p^{2}\right)$, $\mu \notin G F(p), \mu^{2} \in G F(p)$. (Note that $L$ does not depend upon $\mu$.)

Theorem 7. For any prime $p$ such that $p \equiv 7,13(15)$, let $b, c$ be the unique pair of positive integers such that $p=b^{2}+b c+c^{2}, b>c$, and $(b, c)=1$. Then a chiral polytope $\mathscr{P}$ in $\left\langle\{6,3\}_{(b, c)},\{3,5\}\right\rangle^{\text {ch }}$ with automorphism group

$$
A(\mathscr{P}) \cong P S L_{2}\left(p^{2}\right)
$$

exists. 
Proof. By our assumptions about $p, x^{2}+x+1=0(p)$ has a solution, but there is no solution for $x^{2}-x-1 \equiv 0(p)$. Choose $\omega \in \mathbb{Z}_{p}$ such that $\omega^{2}+\omega+1=0$ and $c=b \omega$. We now operate over the field $\mathbb{Z}_{p}[\tau]$. Again, $A$ is the rotation group $A^{+}(\mathscr{P})$ of a chiral or directly regular polytope of type $\{6,3,5\}$. Clearly, the generating matrices are in $G L_{2}\left(\mathbb{Z}_{p}[\tau]\right)$ and therefore we know that $A^{+}(\mathscr{P})$ is a subgroup of $P G L_{2}\left(\mathbb{Z}_{p}[\tau]\right) \cong P G L_{2}\left(p^{2}\right)$. As before, a subgroup of $P G L_{2}\left(\mathbb{Z}_{p}[\tau]\right) \cong P G L_{2}\left(p^{2}\right)$ must be isomorphic to one of the groups enumerated in the proof for Theorem 14. Of these, $A_{4}, S_{4}, A_{5}$ and the dihedral groups can be eliminated in exactly the same way as in the proof for Theorem 14. For the normalizer $H$ of the $p$-Sylow subgroup to contain $[3,5]^{+} \cong A_{5}, 120$ must divide $p^{2}-1$ and thus $p^{2} \equiv 1(120)$. However, $p \equiv 7,13(15)$, so this is not possible. What remain to be considered are $P S L_{2}\left(\mathbb{Z}_{p}\right)$, $P G L_{2}\left(\mathbb{Z}_{p}\right), P S L_{2}\left(p^{2}\right)$, and $P G L_{2}\left(p^{2}\right)$. Of these, $P S L_{2}(p)$ can be rejected as this group would also require that $p^{2} \equiv 1(120)$.

In order to eliminate $P G L_{2}(p)$ we apply Lemma 9 for the fields $\mathbb{Z}_{p}$ and $\mathbb{Z}_{p}[\tau]$. First, we need to coerce the generators into $S L_{2}\left(\mathbb{Z}_{p}[\tau]\right)$. Note that $-\omega$ is a square in $\mathbb{Z}_{p}[\tau],-\omega=\lambda^{2}$ for some $\lambda \in \mathbb{Z}_{p}$. If $p \equiv 1(4)$, then -1 is a quadratic residue modulo $p$ and thus we can choose $\lambda \in \mathbb{Z}_{p}$. If $p \equiv 3(4)$, the equation $\gamma^{2}=-1$ in $\mathbb{Z}_{p}[\tau]$ has solutions $\gamma=a(1-2 \tau)$ with $a \in \mathbb{Z}_{p}$ and $5 a^{2}=-1$; but -1 and 5 are quadratic nonresidues modulo $p$. Hence, the equation $5 a^{2}=-1$ has a solution in $\mathbb{Z}_{p}$. It follows that -1 is a square in $\mathbb{Z}_{p}[\tau]$, and so is $-\omega$. Now the matrices

$$
\alpha_{1}:=\left[\begin{array}{cc}
\lambda & 0 \\
0 & \lambda^{-1}
\end{array}\right]=\frac{1}{\lambda} \sigma_{1}, \quad \alpha_{2}:=\left[\begin{array}{cc}
\omega & -\tau \\
0 & \omega^{-1}
\end{array}\right]=\frac{1}{\omega} \sigma_{2}, \quad \alpha_{3}:=\left[\begin{array}{cc}
\tau & -1 \\
1 & 0
\end{array}\right]=\sigma_{3}
$$

generate a subgroup $G$ of $S L_{2}\left(\mathbb{Z}_{p}[\tau]\right)$. In particular, $\alpha_{3}^{5}=\sigma_{3}^{5}=-I \in G$. Now assume that $A^{+}(\mathscr{P}) \cong P G L_{2}(p)$, of order $p\left(p^{2}-1\right)$. Then, as subgroups of $P G L_{2}\left(\mathbb{Z}_{p}[\tau]\right),\left\langle\alpha_{1}, \alpha_{2}, \alpha_{3}\right\rangle \cong\left\langle\sigma_{1}, \sigma_{2}, \sigma_{3}\right\rangle \cong P G L_{2}(p)$. However, $G$ is a subgroup of $S L_{2}\left(\mathbb{Z}_{p}[\tau]\right)$ containing $-I$, and is thus of order $2 p\left(p^{2}-1\right)$. Now Lemma 9 applies with $i=2$. Set $\mu:=1-2 \tau$ and

$$
\delta:=\left[\begin{array}{cc}
\mu & 0 \\
0 & \mu^{-1}
\end{array}\right]
$$

Then $\mu^{2} \in \mathbb{Z}_{p}$ and $G$ is conjugate in $G L_{2}\left(\mathbb{Z}_{p}[\tau]\right)$ to $L:=\left\langle S L_{2}\left(\mathbb{Z}_{p}\right), \delta\right\rangle$. Thus we have $L=\varphi G \varphi^{-1}$ with $\varphi \in G L_{2}\left(\mathbb{Z}_{p}[\tau]\right)$. Now the trace of a matrix is conjugation invariant, and thus $\operatorname{tr}\left(\varphi \alpha_{3} \varphi^{-1}\right)=\operatorname{tr}\left(\alpha_{3}\right)=\tau$. The matrices in $S L_{2}\left(\mathbb{Z}_{p}\right)$ have trace in $\mathbb{Z}_{p}$ and thus $\varphi \alpha_{3} \varphi^{-1} \notin S L_{2}\left(\mathbb{Z}_{p}\right)$. Since $L$ has twice the order of $S L_{2}\left(\mathbb{Z}_{p}\right)$, this proves that $\varphi \alpha_{3} \varphi^{-1} \in \delta S L_{2}\left(\mathbb{Z}_{p}\right)$. Thus, $\varphi \alpha_{3} \varphi^{-1}=\delta \alpha$ with $\alpha \in S L_{2}\left(\mathbb{Z}_{p}\right)$. However, if

$$
\alpha=\left[\begin{array}{ll}
a & b \\
c & d
\end{array}\right]
$$

then $\operatorname{tr}(\delta \alpha)=a \mu+d \mu^{-1}=(a+d / 5)(1-2 \tau)$, and hence $\operatorname{tr}(\delta \alpha)$ cannot be $\tau$, which is a contradiction. It follows that we cannot have $A^{+}(\mathscr{P})=P G L_{2}\left(\mathbb{Z}_{p}[\tau]\right)$. 
Now the determinants of $\sigma_{1}, \sigma_{2}, \sigma_{3}$ are all squares, and thus $\left\langle\sigma_{1}, \sigma_{2}, \sigma_{3}\right\rangle$ is contained in the subgroup $P S L_{2}\left(\mathbb{Z}_{p}[\tau]\right)$ of $P G L_{2}\left(\mathbb{Z}_{p}[\tau]\right)$. It follows that we must have $A^{+}(\mathscr{P})=P S L_{2}\left(\mathbb{Z}_{p}[\tau]\right)$.

All that remains is to characterize the facets of $\mathscr{P}$. However, $\omega \in \mathbb{Z}_{p}$ and $c=b \omega$. It follows that the facets must be $\{6,3\}_{(b, c)}$, so that $\mathscr{P}$ must be chiral.

Example 3. Consider the case where $m=7,(b, c)=(2,1)$, and $\omega=2$. Then $A(\mathscr{P}) \cong P G L_{2}\left(\mathbb{Z}_{7}[\tau]\right) \cong P S L_{2}(49)$ which has an order of 5880 . Thus,

$\begin{array}{lr}\text { Toroidal facets } & 1,400, \\ \text { Hexagonal faces } & 4,900, \\ \text { Edges } & 58,800, \\ \text { Vertices } & 980 .\end{array}$

Theorem 8. For any $m=p_{1}^{e_{1}} \cdots p_{k}^{e_{k}}$ where each $p_{j} \equiv 1,4,11,14(15)$, with at least one $p_{j} \equiv 11,14(15)$, a directly regular polytope $\mathscr{P}$ in $\left\langle\{6,3\}_{(m, 0)},\{3,5\}\right\rangle$ exists such that

$$
A^{+}(\mathscr{P}) \cong \begin{cases}P S L_{2}\left(\mathbb{Z}_{m}[\omega]\right) & \text { if every } p_{j} \equiv 1,29,41,14(60), \\ P S L_{2}\left(\mathbb{Z}_{m}[\omega]\right) \bowtie C_{2} & \text { otherwise. }\end{cases}
$$

Proof. By our assumptions about $m, x^{2}+x+1 \equiv 0(m)$ does not have a solution, but $x^{2}-x-1 \equiv 0(m)$ does. Let $\tau$ denote a solution for $x^{2}-x-1 \equiv 0(m)$. We now operate over $\mathbb{Z}_{m}[\omega]$. As before, $A$ is the rotation group $A^{+}(\mathscr{P})$ of a chiral or directly regular polytope of type $\{6,3,5\}$. Consider again the generators $\sigma_{1}, \sigma_{2}$, $\sigma_{3}$ as matrices in

$$
\left.L_{2}^{\langle-\omega\rangle} \mathbb{Z}_{m}[\omega]\right)=\left\{S \in G L_{2}\left(\mathbb{Z}_{m}[\omega]\right) \mid \operatorname{det}(S) \in\langle-\omega\rangle\right\}
$$

Then

$$
B:=\sigma_{1}^{4} \sigma_{2} \sigma_{3}=\left[\begin{array}{rr}
0 & -1 \\
1 & 0
\end{array}\right] \text { and } U:=\left(\sigma_{1} \sigma_{2} \sigma_{1}^{3}\right)^{\kappa}=\left[\begin{array}{ll}
1 & 1 \\
0 & 1
\end{array}\right] \text {, }
$$

where $\kappa$ is the multiplicative inverse of $\tau$, and

$$
\sigma_{1}^{4} U \sigma_{1}^{2}=\left[\begin{array}{cc}
1 & \omega \\
0 & 1
\end{array}\right]
$$

Now we can argue as in the proof of Theorem 4. In particular, $A=$ $P L_{2}^{\langle-\omega\rangle}\left(\mathbb{Z}_{m}[\omega]\right)$. However, this group is isomorphic to $P S L_{2}\left(\mathbb{Z}_{m}[\omega]\right)$ if -1 is a square in $\mathbb{Z}_{m}[\omega]$, and to $P S L_{2}\left(\mathbb{Z}_{m}[\omega]\right) \propto C_{2}$ if -1 is a nonsquare. This proves our statement about the group. 
Finally, the ring automorphism $\omega \mapsto \bar{\omega}=\omega^{2}$ induces an involutory group automorphism $\rho$ of $A$ as in (5). Consequently, the resulting polytope is directly regular. In particular, its facets are of type $\{6,3\}_{(m, 0)}$.

Corollary 2. Let $p$ be a prime with $p \equiv 11,14(15)$. Then a directly regular polytope $\mathscr{P}$ in $\left\langle\{6,3\}_{(p, 0)},\{3,5\}\right\rangle$ exists such that

$$
A^{+}(\mathscr{P}) \cong \begin{cases}P S L_{2}\left(p^{2}\right) & \text { if } p \equiv 29,41(60) \\ P G L_{2}\left(p^{2}\right) & \text { otherwise }\end{cases}
$$

Proof. Now $\mathbb{Z}_{p}[\omega]$ is a field of order $p^{2}$. Also, $P G L_{2}(q) \cong P S L_{2}(q) \ltimes C_{2}$ for each $q=p^{k}$.

\section{References}

1. H. S. M. Coxeter, Regular honeycombs in hyperbolic space, in: Twelve Geometric Essays, Southern Illinois University Press, Carbondale, IL, 1968, pp. 199-215.

2. H. S. M. Coxeter, Twisted Honeycombs, Regional Conference Series in Mathematics, American Mathematical Society, Providence, RI, 1970.

3. H. S. M. Coxeter, Regular Polytopes, 3rd edition, Dover, New York, 1973.

4. H. S. M. Coxeter and W. O. J. Moser, Generators and Relations for Discrete Groups, 4th edition, Ergebnisse der Mathematik und Ihrer Grenzgebiet, Vol. 14, Springer-Verlag, Berlin, 1980.

5. L. Danzer and E. Schulte, Reguläre Inzidenzkomplexe I, Geometriae Dedicata 13 (1982), 295-308.

6. D. Garbe, A remark on nonsymmetric compact Riemann surfaces, Archiv der Mathematik 30 (1978), 435-437.

7. B. Grünbaum, Regularity of graphs, complexes and designs, in Problèmes combinatoire et théorie des graphes, Coll. Int. CNRS, Vol. 260, CNRS, Orsay, 1977, pp. 191-197.

8. G. H. Hardy and E. M. Wright, An Introduction to the Theory of Numbers, 5th edition, Oxford University Press, Oxford, 1979.

9. P. McMullen and E. Schulte, Regular polytopes from twisted Coxeter groups, Mathematische Zeitschrift 201 (1989), 209-226.

10. P. McMullen and E. Schulte, Regular polytopes from twisted Coxeter groups and unitary reflexion groups, Advances in Mathematics 82 (1990), 35-87.

11. P. McMullen and E. Schulte, Constructions for regular polytopes, Journal of Combinatorial Theory, Series A 53 (1990), 1-28.

12. P. McMullen and E. Schulte, Hermitian forms and locally toroidal regular polytopes, Advances in Mathematics 82 (1990), 88-125.

13. P. McMullen and E. Schulte, Higher toroidal regular polytopes, Advances in Mathematics (to appear).

14. P. McMullen and E. Schulte, Abstract regular polytopes (monograph in preparation).

15. E. Schulte, Reguläre Inzidenskomplexe II. Geometriae Dedicata 14 (1983), 35-56.

16. E. Schulte, Amalgamation of regular incidence-polytopes, Proceedings of the London Mathematics Society (3) 56 (1988), 303-328.

17. E. Schulte and A. I. Weiss, Chiral polytopes, in: Applied Geometry and Discrete Mathematics (the Klee Festschrift), P. Gritzmann and B. Sturmfels, eds., DIMACS Series in Discrete Mathematics and Theoretical Computer Science, Vol. 4, American Mathematical Society and Association for Computing Machinery, New York, 1991, pp. 493-516.

18. E. Schulte and A. I. Weiss, Chirality and projective linear groups, Discrete mathematics (to appear).

19. H. Schwerdtfeger, Geometry of Complex Numbers, Dover, New York, 1979. 
20. F. A. Sherk, A family of regular maps of type $\{6,6\}$, Canadian Mathematical Bulletin 5 (1962), 13-20.

21. D. M. Y. Sommerville, An Introduction to the Geometry of n Dimensions, Methuen, London, 1929.

22. R. P. Stanley, Enumerative Combinatorics, Volume 1, Wadsworth \& Brooks/Cole, Monterey, CA, 1986.

23. M. Suzuki, Group Theory I, English edition, Springer-Verlag, New York, 1982.

24. A. I. Weiss, Incidence-polytopes with toroidal cells, Discrete \& Computational Geometry 4 (1989), $55-73$.

Received November 18, 1993, and in revised form May 9, 1994. 